\title{
Ethnologies
}

\section{Le musée du quai Branly au miroir de ses prédécesseurs}

\section{Emmanuel Désveaux}

Volume 24, numéro 2, 2002

\section{Musées}

Museums

URI : https://id.erudit.org/iderudit/006648ar

DOI : https://doi.org/10.7202/006648ar

Aller au sommaire du numéro

\section{Éditeur(s)}

Association Canadienne d'Ethnologie et de Folklore

ISSN

1481-5974 (imprimé)

1708-0401 (numérique)

Découvrir la revue

Citer cette note

Désveaux, E. (2002). Le musée du quai Branly au miroir de ses prédécesseurs. Ethnologies, 24(2), 219-227. https://doi.org/10.7202/006648ar

\section{Résumé de l'article}

Les contours du musée d'ethnographie se dessinent au XIX ${ }^{\mathrm{e}}$ siècle, sous l'influence des voyages d'exploration et du mouvement de naturalisation qui caractérisent ce siècle. Commence alors le règne de l'anthropologie physique qui organise le musée d'ethnologie comme une galerie des trophées d'un colonialisme triomphant, parallèlement à la conception évolutionniste de laquelle découle la science raciste qu'est l'anthropométrie. Au début du $\mathrm{XX}^{\mathrm{e}}$ siècle, la technique du diorama sous-tend un nouveau paradigme ethnographique, qui tire en partie sa légitimité de la pratique du terrain de longue durée instiguée par Malinowski ; prétendant à une scientificité supérieure, elle traduit aussi un net progrès moral. Cependant, cette présentation erronée ne rend pas compte des grandes transformations que connaissent ces civilisations au contact de la modernité et conforte le visiteur dans une vision évolutionniste ; la succession des vitrines présente ces populations comme vivant dans un monde séparé. Le nouveau musée qui se construit aujourd'hui entend rompre définitivement avec une présentation évolutionniste devenue immorale et en éradiquer la fiction, en mettant l'accent davantage sur la diversité des cultures et la dimension artistique héritée d'un siècle d'art moderne.
Ce document est protégé par la loi sur le droit d'auteur. L'utilisation des services d'Érudit (y compris la reproduction) est assujettie à sa politique d'utilisation que vous pouvez consulter en ligne.

https://apropos.erudit.org/fr/usagers/politique-dutilisation/ 


\title{
Le musée du quai Branly au miroir de ses prédécesseurs
}

\author{
Emmanuel Désveaux \\ Maitre de conférences à l'EHESS \\ Directeur du projet pour la recherche et \\ l'enseignement au musée du quai Branly, Paris
}

Lactuel grand projet muséal parisien, censé remplacer le musée de l'Homme, ne trouve pas son nom. On l'appelle donc musée du quai Branly, simple toponyme (mais le Louvre aussi est un toponyme puisque c'était le lieu où était installée la louvrerie du roi ${ }^{1}$ ). Cela étant, cette incapacité à s'auto-dénommer est symptomatique car, à bien des égards, il s'agit d'un projet atypique, sur lequel trop de désirs se sont investis, trop d'attentes se sont manifestées, trop de revendications se sont cristallisées. Aujourd'hui, le projet est entré dans une seconde phase, plus opérationnelle; les grues sont à pied d'œuvre sur le site et ce que nous appelons le chantier des collections - le déménagement, le traitement et l'inventaire des collections - est entamé. Pourtant, tout n'est pas réglé, loin de là. À mon sens, pour bien comprendre l'enjeu, il faut voir comment le projet parisien s'inscrit dans le mouvement global de redéfinition du musée d'ethnologie auquel nous assistons tous, mais il nous faut également souligner quelques singularités de notre projet. Singularités typiquement européennes d'abord, notamment par rapport à ce qui se fait ici, singularités françaises ensuite, lesquelles ne sont pas seulement de nature politique mais aussi de nature institutionnelle, donc culturelle car, n'en doutons pas, les institutions d'un pays reflètent son état culturel.

Il nous est inutile de remonter jusqu'au XVI e siècle et au cabinet de curiosités où cohabitaient spécimens naturalistes et artefacts ethnographiques. Rappelons simplement que le modèle du cabinet de

1. Lieu appartenant au domaine du roi, où, avant la construction du château-fort de Philippe-Auguste, était cantonnée la louveterie, compagnie de gens d'armes chargés de traquer les loups. 
curiosités a duré presque trois siècles sans subir beaucoup de changements et que c'est à partir du milieu du XIX ${ }^{e}$ siècle que se dessinent en conséquence les contours du musée d'ethnographie tel que beaucoup d'entre nous l'ont connu, tel que nous le connaissons parfois encore et surtout tel que nous voulons nous en détacher aujourd'hui, en quête que nous sommes d'autres solutions pour donner à voir les autres. Or que se passe-t-il à cette époque? Une multiplication considérable des voyages d'exploration et de découvertes à travers ce monde (qui touche d'ailleurs aussi l'Amérique du Nord) et un vaste mouvement de naturalisation de ce monde. Devant la complexité croissante du monde, le modèle créationniste ne résiste plus. Au fond, c'est cela les Lumières. Or, depuis Linné, naturalisation équivaut à classement. Dès lors que l'ordre de la nature n'est plus d'origine divine, il gît dans les choses elles-mêmes et attend donc son explicitation. L'humain n'échappe pas au mouvement, ce qui explique l'émergence des paradigmes typologiques de l'anthropologie physique, lesquels débouchent en toute logique sur le racisme $e^{2}$. En parallèle, on se pose des questions sur la classification des objets ethnographiques. Si abandonner le modèle du cabinet de curiosités a opéré la première d'entre elles en les séparant des spécimens naturalistes (Thomsen 1937), il reste beaucoup à faire afin d'établir des critères de classement interne aux objets. Il faudra à ce propos entreprendre un travail d'analyse de la correspondance entre Jomard et Siebold. Edmé-François Jomard (1777-1862) n'est pas seulement un spécialiste des antiquités égyptiennes; géographe, il s'intéresse de très près aux collections d'objets " ethnographiques ". C'est d'ailleurs lui qui patronnera Hamy, fondateur du musée d'ethnographie du Trocadéro, lequel est, à Paris, le précurseur immédiat du musée de l'Homme. Quant à Philipp Franz von Siebold (1796-1866), bavarois d'origine et médecin de formation, il séjourne au Japon dans les années 1830 et en rapporte un ensemble important d'objets plusieurs centaines - illustrant la vie quotidienne. Il s'interroge sur la manière la plus opportune de les présenter (Kouwenhoven et al. 2000).

Il est vrai que l'état d'esprit triomphaliste qui accompagne les conquêtes coloniales de la seconde moitié du XIX ${ }^{e}$ siècle va partiellement résoudre la question: les galeries des musées d'ethnographie seront avant tout des galeries de trophées, des endroits où est exposé le butin ramené des lointaines expéditions. Le musée de Tervuren, situé dans la banlieue de Bruxelles, est probablement la

2. Voir à ce propos la belle démonstration de Jacqueline Duvernay-Bollens (1995). 
caricature de cette situation. Le Congo belge était sous la coupe directe du roi Léopold II. Ses réserves contiennent près de 300000 objets ethnographiques (autant que notre futur musée dont les collections «couvrent » l'ensemble de la planète) qui proviennent tous de cette colonie personnelle du roi. Une sorte de vertige saisit le visiteur des réserves de Tervuren au spectacle de ces rangées de masques, de statues, de costumes de même type : il est difficile pour lui de se départir de l'impression que cette zone géographique a été littéralement vidée des éléments matériels de ses cultures, les missionnaires ayant évidemment été les grands complices de cette opération. On conviendra que cet exemple présente un caractère un peu extrême. Rendons-nous maintenant dans de nombreux musées des villes des provinces françaises. On découvrira alors d'appréciables collections d'objets océaniens (Notter 1997). On est frappé de constater que l'essentiel de ces collections consiste en casse-têtes ou en armes. L'indéniable qualité plastique de ce type d'objets ne suffit pas à expliquer leur omniprésence dans les collections. En réalité, s'ils étaient rapportés en nombre par les officiers de marine (lesquels les offraient ensuite au muséum de leur ville natale), c'est précisément parce qu'ils évoquent la relation qui est en train de se nouer entre autochtones et puissances coloniales. Ces armes parlent de combats — que d'ailleurs nul n'eut à mener, à quelques exceptions près - qui ont permis à l'Occident (que ces officiers nonchalands incarnaient) de subjuguer "le sauvage » et d'ouvrir ainsi la voie à son « apprivoisement ». C'était l'époque également, disons-le pour mémoire, des terribles zoos humains, c'est-à-dire de l'installation, derrière des grilles, de groupes d'autochtones.

En France on assiste d'ailleurs à une curieuse entente entre les laïcs et les religieux à propos de ces terrains exotiques. L'arrangement apparaît même contre-nature lorsqu'on sait combien, en cette France de la deuxième moitié du XIX ${ }^{\mathrm{e}}$ siècle, l'antagonisme est profond entre les uns et les autres. Les pères laïcs de la III République ne virent en effet aucun inconvénient à confier "l'éducation des sauvages » aux missionnaires. Mais cette alliance nous révèle l'importance croissante que prend la conception évolutionniste comme paradigme intellectuel dominant. Les religieux acceptent l'idée d'une certaine modernité de l'Occident, les populations exotiques et leurs croyances totémiques, animistes ou autres jouant le rôle des «enfants » de l'humanité, de ceux sur lesquels le devoir de charité impose de se pencher afin de les éclairer sur le vrai Dieu. Quant aux laïcs, ils pensent tout simplement 
qu'accepter l'existence d'un Dieu unique, serait-il en trois personnes, constitue une étape nécessaire avant d'accéder aux vraies lumières de la raison. Cela viendra plus tard, comme la démocratie. Dans la même perspective, bien que sans revenir longuement dessus, rappelons qu'à cette époque l'anthropologie physique, la préhistoire et l'ethnologie cohabitent dans des institutions - musée de l'Homme, Museum of Mankind, etc. - qui elles-mêmes font partie des muséums d'histoire naturelle (Paris, Londres, New York, Ottawa). Parallèlement à la constitution de collections d'objets ethnographiques, on entasse les crânes et on prétend déduire l'intelligence, la place dans l'évolution des uns et des autres d'après leur taille et leur forme respectives. Les pires délires racistes trouvèrent leurs justifications au sein de ces institutions scientifiques. Aucun pays n'a été épargné. Même le grand Boas, champion incontesté de la reconnaissance de l'altérité culturelle, s'était adonné dans sa jeunesse aux plaisirs de l'anthropométrie (Boas 1891).

Au début du $\mathrm{XX}^{\mathrm{e}}$ siècle se met toutefois progressivement en place un nouveau paradigme muséographique. Au lieu de présenter les objets comme des trophées, témoignage de la barbarie des peuplades exotiques, on tend à une reconstitution ethnographique. C'est la technique du diorama. Celle-ci revient en gros à tenter une sorte de restitution ethnographique autour de mannequins mis en situation: costumes, outils, armes, parfois habitation entière, le tout sur un fond peint représentant le paysage, l'environnement naturel de la scène. Aujourd'hui ces reconstitutions nous paraissent dépassées : n'importe quel reportage photographique ou filmé nous apparaît beaucoup plus coloré, vivant, évocateur. De la même façon que nous avions détecté dans l'accumulation des casse-têtes un message implicite sur le supposé combat héroïque de la civilisation contre la barbarie, on peut se demander si le diorama ne traduit pas aussi une nouvelle étape de notre relation à l'altérité. On notera ainsi que l'innovation muséographique accompagne un changement dans les pratiques d'enquête de l'anthropologie qui prône désormais le terrain dit ethnographique. Il ne s'agit plus de rapporter un trophée d'une expédition lointaine mais de transporter en bloc une tranche d'existence exotique dont l'ethnologue-muséographe - qui l'aurait lui-même vécue sur le terrain — est habilité à garantir l'authenticité. Nous sommes dans la fiction la plus complète, bien entendu: le plus souvent les différents vêtements dont sont affublés les mannequins viennent de parures distinctes. 
L'exigence muséographique impose aussi une sorte de maximalisation de la présentation : le spectateur verra donc un néo-guinéen en grand costume de rituel s'adonner à la taille d'une hache, ou au sarclage de son champ, etc. Cette présentation muséographique tire une partie de sa légitimité de la pratique du terrain de longue durée qui, sous l'influence de Malinowski, tend à devenir à cette époque la norme, aussi bien en Europe qu'en Amérique du Nord. Bien qu'aujourd'hui nous ayons plus de difficultés à le percevoir, cette muséographie, qui prétend à un statut de scientificité supérieure par rapport à celles qui la précédaient, traduit aussi un progrès moral (sinon scientifique) par rapport à la situation antérieure. On s'efforce de restituer un mode de vie dans son ensemble, autrement dit dans une totalité culturelle. À travers cette dernière, on confère une dignité aux autres. Nous avons tous en tête le ridicule de ces reconstitutions, ou à tout le moins l'impression de malaise qu'on ressent devant cette pseudo-réalité : l'idéalisation platonicienne qui est derrière elle produit une représentation singulièrement figée. D'ailleurs, bien avant que la question de la restitution n'occupe l'essentiel des débats, c'est pour dénoncer ce type de présentations que des voix autochtones se sont élevées au sujet du musée d'ethnographie classique. Au fond, les personnes concernées - les sujets de ces mises en scène sclérosées — voyaient mieux que nous combien ce dispositif s'inscrivait dans la continuité du zoo humain. Bien sûr, autre défaut de cette mise en scène qui correspond d'ailleurs au péché le plus grave de l'anthropologie de cette époque — du moins si l'on en croit les actes du procès que les postmodernistes lui ont instruit (Clifford 1988) - ces présentations étaient entièrement a-historisées, faisant cohabiter dans les mêmes vitrines et sous le même discours des objets appartenant à des époques distinctes. La représentation " ethnographique » se donne à voir dans les grands musées occidentaux à l'époque même où les groupes concernés commencent à subir, au contact de la modernité, de grandes transformations, autrement dit au moment où l'aspect traditionnel de ces cultures tend à s'éroder très rapidement. Processus sur lequel, bien sûr, la muséographie restait muette. On observe le même décalage qu'à la période précédente qui rapporte en masse des casse-têtes océaniens, entretenant l'illusion que la conquête des sauvages avait été une prouesse militaire ponctuée de corps à corps héroïques, alors qu'elle s'est faite au pire à coups de canonnière, rendant bien dérisoires sur le terrain les redoutables cassetêtes rapportés comme trophées. 
Il est un second défaut inhérent à ces dispositifs, lequel nous semble avoir été négligé par les contempteurs du musée d'ethnologie classique et qui mérite pourtant d'être souligné. Les dioramas privilégient les scènes de la «vie quotidienne ». Cela tient en partie à des raisons de commodité. Nul doute qu'en l'absence des puissants moyens de reproduction dont nous disposons aujourd'hui, l'ethnographemuséographe éprouve des scrupules à présenter un rituel dont il connait, par expérience, l'extrême complexité. Mais cela tient également à des raisons théoriques. Une mise en scène suppose une certaine matérialité. L'ethnographe serait-il fonctionnaliste, il inclinera à présenter des réalités substantielles. Notons que la problématique de la production matérielle devenait la question dominante au sein de la discipline, que ce soit dans sa version typiquement américaine, à savoir l'écologisme culturel, ou dans de multiples versions européennes, plus ou moins influencées par le marxisme. Nous sommes désormais à une époque où l'anthropologie a atteint sa maturité ; elle a pris sans ambiguïté toutes les distances nécessaires avec l'évolutionnisme primaire, teinté de racisme, de ses origines. Il n'empêche qu'à travers la reconstitution des modes de vie exotiques, c'est le développement technique qui continue à servir de fondement à la muséographie. En dépit des bonnes intentions du muséographe, la vitrine dans laquelle tel chasseur de caribou s'apprête à flécher sa proie sur fond de paysage désolé des barren grounds, dans laquelle telle tisserande andine engage sa navette dans un grossier métier, dans laquelle tel forgeron africain semble peiner à porter le métal à son point de fusion, ne pouvait échapper à la lecture en termes de progrès technique de la part du visiteur non averti, lequel entretenait encore bien souvent à l'époque, dans l'exercice quotidien de sa profession, un rapport physique avec la production. Le musée d'ethnographie classique renvoie à un évolutionnisme spontané qui caractérise nécessairement le sens commun d'une société foncièrement tournée vers le progrès technique. La hiérarchie des cultures, qu'animé des meilleurs sentiments on avait voulu évacuer par la porte, entre par la fenêtre. Pourtant, du point de vue "scientifique », cette muséographie présente toutes les vertus puisqu'en présentant chaque culture ou chaque société en tant qu'unité cohérente, on défend l'idée que ses membres vivaient dans un monde qui leur était propre : ils ne perçoivent pas le stade inférieur de leur développement technique. L'anthropologie laissait entendre que chaque société construit autour de ses membres une sorte de "prison dorée » culturaliste. L'incongruité d'une telle option intellectuelle apparaît de façon encore plus patente à propos de l'Amérique, et plus 
encore de l'Amérique du Nord: la succession des vitrines va refléter un découpage en aires culturelles à la Kroeber, sans qu'il soit jamais remis en question, alors que ce dernier nous apparaît de plus en plus hautement discutable (Désveaux 2001, 2002).

Aujourd'hui donc se construit un nouveau musée à Paris. Pas entièrement nouveau bien sûr, car les collections qui lui serviront d'assise existent déjà, bien qu'elles seront sensiblement enrichies et complétées par rapport à ce que possédait le département d'ethnologie du musée de l'Homme et le musée national des arts d'Afrique et d'Océanie (MNAAO). En tout cas, une nouvelle localisation et un nouveau bâtiment, dû à l'architecte Jean Nouvel. En quoi la muséographie devrat-elle être nouvelle, innover? Selon nous, elle doit répondre à deux objectifs. Premier objectif : larguer définitivement les amarres avec l'évolutionnisme devenu intolérable d'un point de vue moral, ou si l'on préfère, du point de vue du public, alors même qu'il garde de très honorables défenseurs du point de vue scientifique. A cet égard, fonder un nouveau musée avec une administration propre, affranchie de la tutelle institutionnelle du muséum d'histoire naturelle, constitue un pas de nature politique important. Rendons hommage au Canada qui avait amorcé le mouvement, il y a maintenant une vingtaine d'années, en dépouillant d'une partie de ses collections son musée de l'Homme à Ottawa pour créer à Hull, un peu plus loin, de l'autre côté de la rivière, le musée des Civilisations. Le deuxième objectif est d'éviter que le musée d'ethnologie soit un lieu de fiction, comme il l'a été tour à tour du temps de la muséographie «trophée » et la muséographie « ethnographique». Sinon il sera toujours le lieu d'une fiction. Or, précisément, il nous semble que, dans un univers culturel de plus en plus forgé par la fiction, car de plus en plus soumis aux diktats du marché (la fiction, compte tenu de sa flexibilité intrinsèque possède un potentiel de séduction, donc marchand, beaucoup plus élevé que la réalité), le rôle des grandes institutions d'état est de résister. Ainsi, par exemple, s'il nous paraît important d'en tenir compte, nous ne pensons pas judicieux de construire un discours autour des collections qui réponde exclusivement aux revendications des autochtones. Car celles-ci s'avèrent instables, peu compatibles les unes avec les autres, ni d'ailleurs avec l'état des connaissances scientifiques. Mieux, leurs racines idéologiques, pour légitimes qu'elles soient, tendent à filtrer les réalités de l'histoire. Leur accorder une trop grande importance risque en effet de nous faire retomber dans la fiction. 
Nous sommes conscient que le pari de cette nouvelle muséographie à inventer n'est pas aisé à gagner. La voie est étroite qui choisit de mettre en avant la diversité des cultures et l'accent sur l'art. Magnifier la diversité culturelle entre en résonance avec l'impératif de l'altérité qui conditionne notre vision du monde. Parallèlement, la dimension esthétique des sociétés apparaît aujourd'hui totalement déconnectée de leur technique et en fait un vecteur idéal d'un message en apparence profondément anti-évolutionniste. Une écorce peinte aborigène rivalise avec un masque de la côte Nord-Ouest, qui lui-même vaut un reliquaire du Zaïre, qui à son tour vaut un chapiteau roman, etc. Mais, cette leçon, nous la devons à un siècle "d'art moderne » et de pratiques esthétiques qui valorisent l'intention, la forme et ses significations, de préférence sur le mode de la connotation, au détriment de la facture. Nous la devons à des édicteurs de (bon) goût, ainsi que l'ont montré d'innombrables travaux à ce sujet dus à Bourdieu et à son école (Bourdieu 1979). Aussi, malgré tous nos efforts afin d'être le plus lucide possible, en particulier grâce à l'examen critique du travail de nos prédécesseurs, il se pourrait que nos options muséographiques aient leurs contre-effets, et en particulier celui-ci : comment se fait-il que ces gens-là soient d'aussi grands artistes et qu'ils ne s'en soient pas rendu compte ? En définitive, ils nous doivent leur consécration à ce titre... Ils la doivent au musée en tant qu'institution. Alors, du zoo humain à la vitrine ethnographique, du casse-tête trophée au masque rituel transfiguré en chef-d'œuvre de la sculpture, les choses seraient toujours les mêmes. Mais peut-il en être autrement, sauf à abolir la grande institution muséale d'Etat, sauf à tout restituer, mais du coup à accélérer la victoire absolue du marché ? Car, n'en doutons pas, dans ce dernier cas, ce seront les marchands qui in fine rafleront la mise. 


\section{Références}

Boas, Franz, 1891, "Physical Characteristics of the Indians of the North Pacific Coast », American Anthropologist 4(1) : 25-35.

Bourdieu, Pierre, 1979, La Distinction, critique sociale du jugement, Paris, Éditions de Minuit.

Clifford, James, 1988, The Predicament of Culture, Twentieth-Century Ethnography, Literature, and Art, Cambridge, Harvard University Press.

Désveaux, Emmanuel, 2001, Quadratura americana, essai d'anthropologie lévi-straussienne, Genève, Georg. (dir.), 2002, Kodiak, Alaska, Paris, Adam Biro et Musée du quai Branly (catalogue de l'exposition éponyme).

Duvernay-Bollens, Jacqueline, 1995, Les Géants patagons: Voyage aux origines de l'homme, Paris, Éditions Michalon.

Kouwenhoven, Arlette, Matthi Forrer et Mark Poysden, 2000, Siebold and Japan, Leyde, Hotei.

Notter, Annick (dir.), 1997, La découverte du paradis, Océanie. Curieux, navigateurs et savants, Paris, Somogy.

Thomsen, Thomas, décembre 1937, « The Study of Man, Denmark organized the World's First Ethnographical Museum », The AmericanScandinavian Review: 309-318. 